\title{
Molecular composition of the peri-islet basement membrane in NOD mice: a barrier against destructive insulitis
}

\author{
H. F. Irving-Rodgers • A. F. Ziolkowski • C. R. Parish • \\ Y. Sado $\cdot$ Y. Ninomiya $\cdot$ C. J. Simeonovic $\cdot$ R. J. Rodgers
}

Received: 28 January 2008 /Accepted: 19 May 2008 / Published online: 17 July 2008

(C) The Author(s) 2008

\begin{abstract}
Aims/hypothesis This study examined whether the capsule which encases islets of Langerhans in the NOD mouse pancreas represents a specialised extracellular matrix (ECM) or basement membrane that protects islets from autoimmune attack.

Methods Immunofluorescence microscopy using a panel of antibodies to collagens type IV, laminins, nidogens and perlecan was performed to localise matrix components in NOD mouse pancreas before diabetes onset, at onset of diabetes and after clinical diabetes was established (28.5 weeks post-onset).

Results Perlecan, a heparan sulphate proteoglycan that is characteristic of basement membranes and has not previously been investigated in islets, was localised in the peri-islet capsule and surrounding intra-islet capillaries. Other components present in the peri-islet capsule included laminin chains $\alpha 2, \beta 1$ and $\gamma 1$, collagen type IV $\alpha 1$ and $\alpha 2$, and nidogen 1
\end{abstract}

H. F. Irving-Rodgers $\cdot$ R. J. Rodgers $(\bowtie)$

Research Centre for Reproductive Health, Department of

Obstetrics and Gynaecology, The University of Adelaide,

Adelaide SA 5005, Australia

e-mail: ray.rodgers@adelaide.edu.au

A. F. Ziolkowski • C. R. Parish • C. J. Simeonovic

The Division of Immunology and Genetics, The John Curtin

School of Medical Research, The Australian National University,

Canberra, ACT, Australia

Y. Sado

Division of Immunology, Shigei Medical Research Institute,

Okayama, Japan

\section{Y. Ninomiya}

Department of Molecular Biology and Biochemistry,

Okayama University Medical School,

Okayama, Japan and 2. Collagen type IV $\alpha 3-\alpha 6$ were not detected. These findings confirm that the peri-islet capsule represents a specialised ECM or conventional basement membrane. The islet basement membrane was destroyed in islets where intraislet infiltration of leucocytes marked the progression from non-destructive to destructive insulitis. No changes in basement membrane composition were observed before leucocyte infiltration.

Conclusions/interpretation These findings suggest that the islet basement membrane functions as a physical barrier to leucocyte migration into islets and that degradation of the islet basement membrane marks the onset of destructive autoimmune insulitis and diabetes development in NOD mice. The components of the islet basement membrane that we identified predict that specialised degradative enzymes are likely to function in autoimmune islet damage.

Keywords Autoimmunity - Basement membrane . Collagen · Islets · Laminin · Matrix · Nidogen · NOD ·

Perlecan

$\begin{array}{ll}\text { Abbreviations } \\ \text { APC } & \text { antigen-presenting cell } \\ \text { ECM } & \text { extracellular matrix } \\ \text { EHS } & \text { Engelbreth-Holm-Swarm tumour } \\ \text { HS } & \text { heparan sulphate } \\ \text { HSPG } & \text { heparan sulphate proteoglycan } \\ \text { MNC } & \text { mononuclear cell }\end{array}$

\section{Introduction}

In humans and in NOD mice, type 1 diabetes results from autoimmune destruction of the insulin-secreting beta cells of the islets of Langerhans in the pancreas. In NOD mice, 
the autoimmune response occurs in two stages. The first stage appears soon after weaning and is a peri-islet insulitis where mononuclear cells (MNCs) accumulate around the periphery of the islets but do not invade the islets. This response has become known as 'non-destructive' or 'benign' autoimmunity. Initially, the insulitis consists of antigen-presenting cells (APCs) such as macrophages and dendritic cells and is subsequently followed by CD4 T cells, CD8 $\mathrm{T}$ cells and $\mathrm{B}$ cells [1]. This non-destructive phase progresses to the second state, destructive autoimmunity, as the mice age, resulting in clinical diabetes when the insulin content of the pancreas declines to $<10 \%$ that of normal mice [2]. It is thought that activation of autoreactive T cells occurs following interaction with APCs that have processed beta cell-derived antigens. Such T cell activation probably occurs locally in the pancreatic lymph nodes and results in the ability of the autoreactive $\mathrm{T}$ cells to migrate through tissues [3]. This involves extravasation through the blood vessel wall and passage through the underlying subendothelial basement membrane and surrounding extracellular matrix $(\mathrm{ECM})$. It would appear that extravasation takes place at least preferentially from the peri-islet capillaries and not the intra-islet capillaries, since intercellular adhesion molecule 1 (ICAM1) is upregulated in these vessels [4] and the amount of peri-islet vasculature actually increases, unlike the intra-islet vascular bed which decreases [5]. Thus, to achieve entry into islets, migrating leucocytes would need to cross any matrix which surrounds and delineates individual islets.

Previous studies of ECM components surrounding pancreatic islets have generated some confusion and this problem appears to have arisen from a limited range of available reagents and thus incomplete analyses. van Deijnen et al. [6] reported the presence of a peri-islet capsule containing collagen type IV and laminin, which was continuous in canine islets, discontinuous in the rat and human, and fragmentary in the case of porcine islets. Jiang et al. [7] concluded that the murine pancreatic islets are not surrounded by a basement membrane. A detailed study by Miner et al. [8] focusing on exocrine acinar tissue identified laminin chains in the murine pancreas, but did not report any laminin staining of islets. Pavin et al. [9] localised laminin around the islets of NOD mice and found disruption of the peri-islet basement membrane associated with intense infiltration by inflammatory cells. Fibronectin is a major ECM protein, which has also been identified around the periphery of islets in the NOD neonatal pancreas [10]. Transplantation studies of islets have shown that for them to survive, ECM is required for islet cell attachment $[11,12]$, suggesting that the matrix is also important for the preservation of islet integrity following isolation.

Our study sought: (1) to clarify the status of the peri-islet capsule in the murine pancreas by means of an exhaustive analysis of the matrix components; (2) to monitor any changes to the composition during the development of diabetes in NOD mice; and consequently (3) to ascertain whether the immunological destruction of islets in situ is likely to require the production by autoimmune leucocytes of specific enzymes capable of degrading matrix components.

\section{Methods}

Experimental animals $\mathrm{NOD} / \mathrm{Lt}$ and $\mathrm{BALB} / \mathrm{c}$ mice were obtained from the Animal Services Division, the John Curtin School of Medical Research, The Australian National University, Canberra, ACT, Australia. NOD/Lt female mice $\geq 24$ weeks of age were killed before or after diabetes onset. Pancreas samples were taken from female NOD/Lt prediabetic mice at 24 to 25.5 weeks of age $(n=5)$ and at 34 to 36.5 weeks of age $(n=5)$. Pancreas was removed from diabetic female NOD/Lt mice on the day clinical diabetes was confirmed (32-49 weeks of age) $(n=5)$, at 2 weeks $(n=6)$ and at 7 to 8.5 weeks after diabetes onset $(n=2)$. Non-fasting blood glucose levels in tail vein blood were measured using a Medisense 2 glucometer. Clinical diabetes was determined to have developed when non-fasting blood glucose levels were $\geq 19 \mathrm{mmol} / \mathrm{l}$. Diabetic mice (unless killed at the time of diabetes onset) were maintained on 2 to $4 \mathrm{U} /$ day s.c. insulin (Humulin Ultralente; Eli Lilly Australia, West Ryde, NSW, Australia). Pancreas samples were also taken from 21-day-old female BALB/c mice $(n=3)$. All experimental procedures on animals were approved by the Australian National University Animal Experimentation Ethics Committee and were in accordance with Principles of laboratory animal care (NIH publication no. 85-23, revised 1985; http://grants1.nih.gov/ grants/olaw/references/phspol.htm).

Immunohistochemistry and histology Pancreas samples from NOD/Lt mice were divided into segments, snapfrozen in liquid Forane (134a; Elf Aochem, Sydney, NSW, Australia) pre-equilibrated with liquid nitrogen and embedded in optimal cutting temperature embedding compound (Sakura Finetechnical, Tokyo, Japan). Frozen tissue sections were used to localise matrix components by an indirect immunofluorescence method [13]. Tissue sections $(10 \mu \mathrm{m})$ were prepared using a CM1800 Leica cryostat (Adeal, Altona North, VIC, Australia), collected on glass slides treated with $0.01 \%$ (wt/vol.) poly l-ornithine hydrobromide (Sigma Chemical, Castle Hill, NSW, Australia) and stored at $-20^{\circ} \mathrm{C}$ until use. Table 1 summarises the antibodies used for immunohistochemistry and the relevant fixation conditions. Unfixed sections were dried under vacuum for $5 \mathrm{~min}$, followed either by fixation in $100 \%$ acetone, $100 \%$ ethanol, formalin or left unfixed (Table 1). Sections were then rinsed in $3 \times 5$ min changes of 
Table 1 Primary antibodies and fixation conditions used for immunohistochemistry

\begin{tabular}{|c|c|c|c|c|c|}
\hline \multirow[t]{2}{*}{ Antigen (species) } & \multicolumn{2}{|c|}{ Primary antibody } & \multirow[t]{2}{*}{ Source [reference] } & \multirow[t]{2}{*}{ Dilution } & \multirow[t]{2}{*}{ Fixation } \\
\hline & Host species & Code & & & \\
\hline Laminin EHS (mouse) & Rabbit & L9393 & Sigma Chemical, Castle Hill, NSW, Australia & $1: 100$ & $100 \%$ ethanol \\
\hline Laminin $\alpha 2$ (mouse) & Rat & $4 \mathrm{H} 8-2$ & Sigma Chemical & $1: 3200$ & Formalin \\
\hline Laminin $\beta 1$ (mouse) & Rat & LT3 & Upstate Biotechnologies, NY, USA & $1: 50$ & Unfixed \\
\hline Laminin $\gamma 1$ (mouse) & Rat & 05-206 & Upstate Biotechnologies & $1: 125$ & Unfixed \\
\hline $\begin{array}{l}\text { Type IV collagens } \alpha 1-\alpha 6 \\
\text { (human) }\end{array}$ & Rat & & Y. Sado ${ }^{a}, Y$. Ninomiya ${ }^{b}[31]$ & $1: 100$ & $100 \%$ acetone \\
\hline $\begin{array}{l}\text { Type IV collagen } \\
\text { (mouse EHS tumour) }\end{array}$ & Rabbit & $2150-1470$ & AbD Serotec, Oxford, UK & $1: 500$ & Formalin \\
\hline Nidogen-1 (mouse) & Rabbit & 914 & M. Dziadek ${ }^{\mathrm{c}}[32]$ & $1: 200$ & Unfixed \\
\hline Nidogen-2 (mouse) & Rabbit & 1130 & T. Sasaki ${ }^{\mathrm{d}}$ & $1: 800$ & Unfixed \\
\hline Perlecan (mouse) & Rabbit & 906 & M. Dziadek ${ }^{\mathrm{c}}$ & $1: 800$ & Unfixed \\
\hline
\end{tabular}

${ }^{a}$ Y. Sado, Division of Immunology, Shigei Medical Research Institute, Okayama, Japan

${ }^{\mathrm{b}}$ Y. Ninomiya, Department of Molecular Biology and Biochemistry, Okayama University Medical School, Okayama, Japan

${ }^{\mathrm{c}}$ M. Dziadek, Garvan Institute of Medical Research, Darlinghurst, NSW, Australia

${ }^{\mathrm{d}}$ T. Sasaki, Max-Plank Institute for Biochemistry, Martinsried, Germany

hypertonic phosphate-buffered saline $(10 \mathrm{mmol} / \mathrm{l}$ sodium/ potassium phosphate with $0.274 \mathrm{~mol} / \mathrm{l} \mathrm{NaCl}$ and $5 \mathrm{mmol} / \mathrm{l}$ $\mathrm{KCl}$; $\mathrm{pH}$ 7.2) before treatment with blocking solution $[10 \%$ (vol./vol.) normal donkey serum] (Sigma Chemical) in antibody diluent containing $0.55 \mathrm{~mol} / \mathrm{l}$ sodium chloride and $10 \mathrm{mmol} / 1$ sodium phosphate [pH 7.1] for $20 \mathrm{~min}$ at room temperature). Incubation with primary antibodies was carried out overnight at room temperature. Secondary antibodies used were either biotin-SP-conjugated AffiniPure donkey anti-rat IgG (1:100; cat. no. 712-066-153) or biotin-SP-conjugated AffiniPure donkey anti-rabbit IgG (1:200; cat. no. 711-066-152), followed by Cy3-conjugated streptavidin (1:100; cat. no. 016-160-084), all from Jackson ImmunoResearch Laboratories (West Grove, PA, USA). For dual localisation secondary antibodies were donkey anti-rat IgG conjugated to Cy3 (1:100; cat. no. 715-165153) and biotin-SP-conjugated AffiniPure donkey antirabbit IgG followed by fluorescein conjugated streptavidin (1:100; cat. no. 016-090-084), also from Jackson. For some dual localisation procedures, sections were also treated with the nuclear stain DAPI solution (Molecular Probes, Eugene, OR, USA). Sections were mounted in mounting medium for fluorescence (Dako Corporation, Carpinteria, CA, USA) and observed and photographed with an Olympus BX50 microscope with an epifluorescence attachment and a Spot RT digital camera (Diagnostic Instruments, Sterling Heights, MI, USA). For light microscopy, frozen sections were fixed in formalin and stained with haematoxylin and eosin. Pancreas tissue from BALB/c mice was fixed in $2.5 \%$ (vol./vol.) glutaraldehyde in $0.1 \mathrm{~mol} / 1$ phosphate buffer. After washes with $\mathrm{H}_{2} \mathrm{O}(3 \times 5 \mathrm{~min})$, tissues were dehydrated in increasing concentrations of acetone [50, 70, 90,95 and $4 \times 100 \%$ (vol./vol.)] at $4^{\circ} \mathrm{C}$ and infiltrated with epoxy resin overnight at room temperature prior to embedding in fresh resin and polymerising overnight at $60^{\circ} \mathrm{C}$. Sections for light microscopy $(1 \mu \mathrm{m})$ were stained with $1 \%$ (wt/vol.) aqueous methylene blue and viewed as above.

\section{Results}

In this study pancreas samples were taken from female NOD/Lt prediabetic mice at 24 to 25.5 weeks of age $(n=5)$ and 34 to 36.5 weeks of age $(n=5)$. Pancreas was also removed from diabetic female NOD/Lt mice on the day that clinical diabetes was confirmed (32-49 weeks of age) $(n=$ $5)$ and at 2 weeks $(n=6)$ and 7 to 8.5 weeks after diabetes onset $(n=2)$. Histological examination of the pancreas from both prediabetic and post-diabetes onset animals revealed that the pancreatic islets were separated from the adjacent exocrine acinar cells by stromal cells and an ECM (Fig. 1a). Immunohistochemical localisation identified the ECM as containing basement membrane components. The basement membrane completely enclosed individual islets and in places was closely apposed to the basement membrane underlying acinar cells (Fig. 1b). The peri-islet basement membrane was immunopositive for at least one of the basement membrane components of the laminin chains of Engelbreth-Holm-Swarm (EHS) tumour $(\alpha 1, \beta 1, \gamma 1)$ (Fig. 1b), laminin $\alpha 2$ (Fig. 2c), laminin $\beta 1$ (Fig. 3b), collagen type IV $\alpha 1$ (Fig. 4a) and $\alpha 2$, laminin $\gamma 1$ (Fig. 5b) perlecan (Fig. 5e), nidogen-1 (Fig. 3c) and nidogen-2 (Fig. 3d), but not collagen type IV $\alpha 3-\alpha 6$. In the adjacent exocrine tissue, acinar basement membranes contained the same components as the peri-islet basement membrane. 

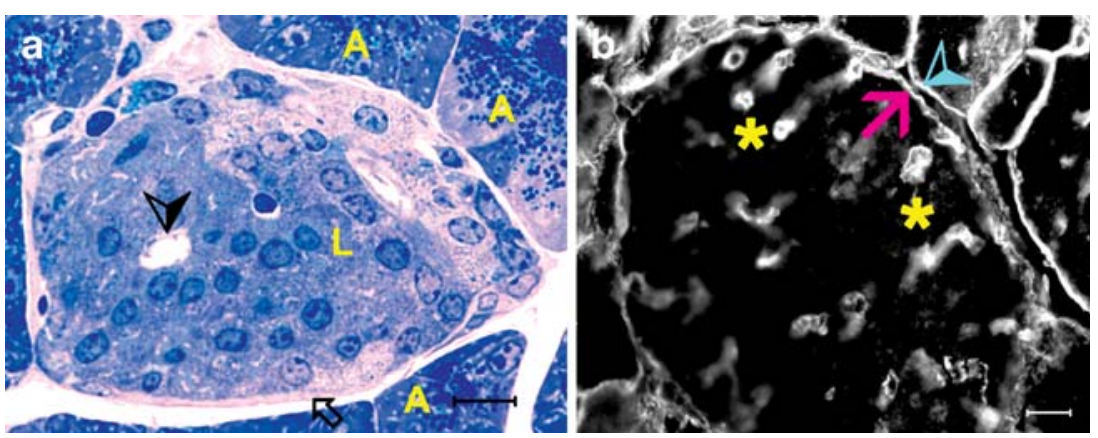

Fig. 1 Morphology of the non-diabetic pancreas (a) and characterisation of the ECM associated with pancreatic islets of Langerhans of prediabetic NOD/Lt mice. The endocrine islet of Langerhans is separated from the adjacent exocrine acinar cells by stromal cells and an ECM. Epoxy resin section of 21-day-old BALB/c mouse pancreas stained with methylene blue. A, exocrine acinar cells; L, islet of Langerhans; arrow, stromal cells and an ECM; arrowhead, capillary within the islet. (b) Immunolocalisation with anti-EHS laminin. The peri-insular basement membrane (arrow) is sometimes in close apposition to the basement membrane of acinar cells (arrowhead) in prediabetic NOD/Lt mouse pancreas aged 34.5 weeks old. Capillaries $\left(^{*}\right)$ are present within the islet. Scale bars (a) $10 \mu \mathrm{m}$, (b) $20 \mu \mathrm{m}$
Within the islet, sub-endothelial basement membranes were also immunopositive for the same components as the periislet basement membrane.

In both prediabetic mice (Figs 2b, 4b, 5c) and diabetic mice (Fig. 5f) leucocyte invasion into islets was observed histologically. This MNC infiltration was associated with loss of peri-islet basement membrane as detected by loss of immunostaining for laminin chains $\alpha 2$ (Fig. 2d), $\beta 1$ and $\gamma 1$ (Fig. 5d), collagen type IV $\alpha 1$ (Fig. 4c) and $\alpha 2$, nidogen-1 and -2 , and perlecan (Fig. 5f). Islets without $\mathrm{MNC}$ infiltration showed no reduction in immunostaining of peri-islet basement membrane components. In contrast to the changes in composition of the peri-islet basement membrane in the presence of destructive insulitis, acinar basement membranes and the sub-endothelial basement membrane of capillaries within the islets remained immunopositive for basement membrane components (Figs 4c, 5d,f). These results indicate that destructive insulitis is selectively associated with complete destruction of the peri-islet basement membrane but not that of other adjacent basement membranes of acini, capillaries or intact islets without MNC infiltration.

\section{Discussion}

This study has demonstrated that pancreatic islets in situ have an uninterrupted boundary or peri-islet capsule, which consists of laminin chains $\alpha 2, \beta 1$ and $\gamma 1$, collagen type IV $\alpha 1$ and $\alpha 2$, perlecan, nidogen- 1 and nidogen-2. Despite the controversy about whether a basement membrane surrounds islets of Langerhans, the previous localisation of collagen type IV [6] suggested that the matrix around islets was a basement membrane. Our novel finding that perlecan, a heparan sulphate (HS) proteoglycan (HSPG) characteristic of basement membranes [14], is present in the islet pericapsule in mice has not been reported previously. Together, with the localisation of laminins $(\alpha 2, \beta 1$ and $\gamma 1)$, collagen type IV ( $\alpha 1$ and $\alpha 2$ ), and nidogen-1 and -2 , the localisation of perlecan provides strong evidence that islets are surrounded by a basement membrane. In addition, it was recently shown that the islet basement membrane of the human pancreas contains laminin $\alpha 5, \beta 1, \beta 2$ (at low level) and $\gamma 1$, while in the mouse laminin $\alpha 5$ (other laminin components were not examined) was irregularly distributed around the islets [15].

In general, basement membranes are thin sheets of ECM that underlie epithelial and endothelial cells and surround individual cells or groups of cells [16, 17], providing physical stability and acting as a barrier to cell invasion [18]. Islets are not regarded as epithelia and by having intra-islet vasculature, they differ from epithelia, even stratified epithelia. However, islet cells have the capacity to form epithelial-like structures in vitro when cultured in gels of collagen I or the laminin 111-rich Matrigel [19]. Recent reports have indicated that islet ECM is laid down by associated vascular endothelial cells [20, 21]. These findings suggest that it would be feasible for islets in vivo to have a surrounding basement membrane. There has been some confusion in the literature as to the existence of a peri-islet basement membrane, due, in particular, to reports of discontinuous staining of basement membrane components around the islet periphery [6, 10, 22]. Additional factors confounding previous interpretations have included incomplete analyses resulting from a limited range of basement membrane-specific reagents, the close proximity of the acinar basement membrane and the presence of subendothelial basement membranes of the vasculature. The present study localised a broad range of individual basement membrane components and also observed islets before and after lymphocyte infiltration where the basement membrane was observed to be present or absent, respectively. In doing so, evidence was found that an islet basement 
Fig. 2 Haematoxylin and eosinstained sections $(\mathbf{a}, \mathbf{b})$ of same islets $(\mathbf{c}, \mathbf{e}, \mathbf{g} ; \mathbf{d}, \mathbf{f}, \mathbf{h})$, respectively, in which laminin $\alpha 2$ (red) $(\mathbf{c}, \mathbf{d})$ and collagen type IV (green) $(\mathbf{e}, \mathbf{f})$ to pancreases of NOD/Lt mice ( 25 weeks), with $(\mathbf{d}, \mathbf{f})$ or without $(\mathbf{c}, \mathbf{e})$ insulitis were co-localised. $(\mathbf{g}, \mathbf{h})$

Merged images of laminin $\alpha 2$ (c, d) with collagen type IV (e, f) respectively. Nuclei are counterstained blue with DAPI. Star, leucocyte infiltration. Laminin $\alpha 2(\mathbf{c}, \mathbf{d})$ and collagen type IV $(\mathbf{e}, \mathbf{f})$ are immunolocalised to the basement membrane underlying acinar cells (arrowheads) and sub-endothelial basement membrane $(*)$, and to the peri-islet basement membrane in the absence of insulitis (arrows). Scale bar, $10 \mu \mathrm{m}$
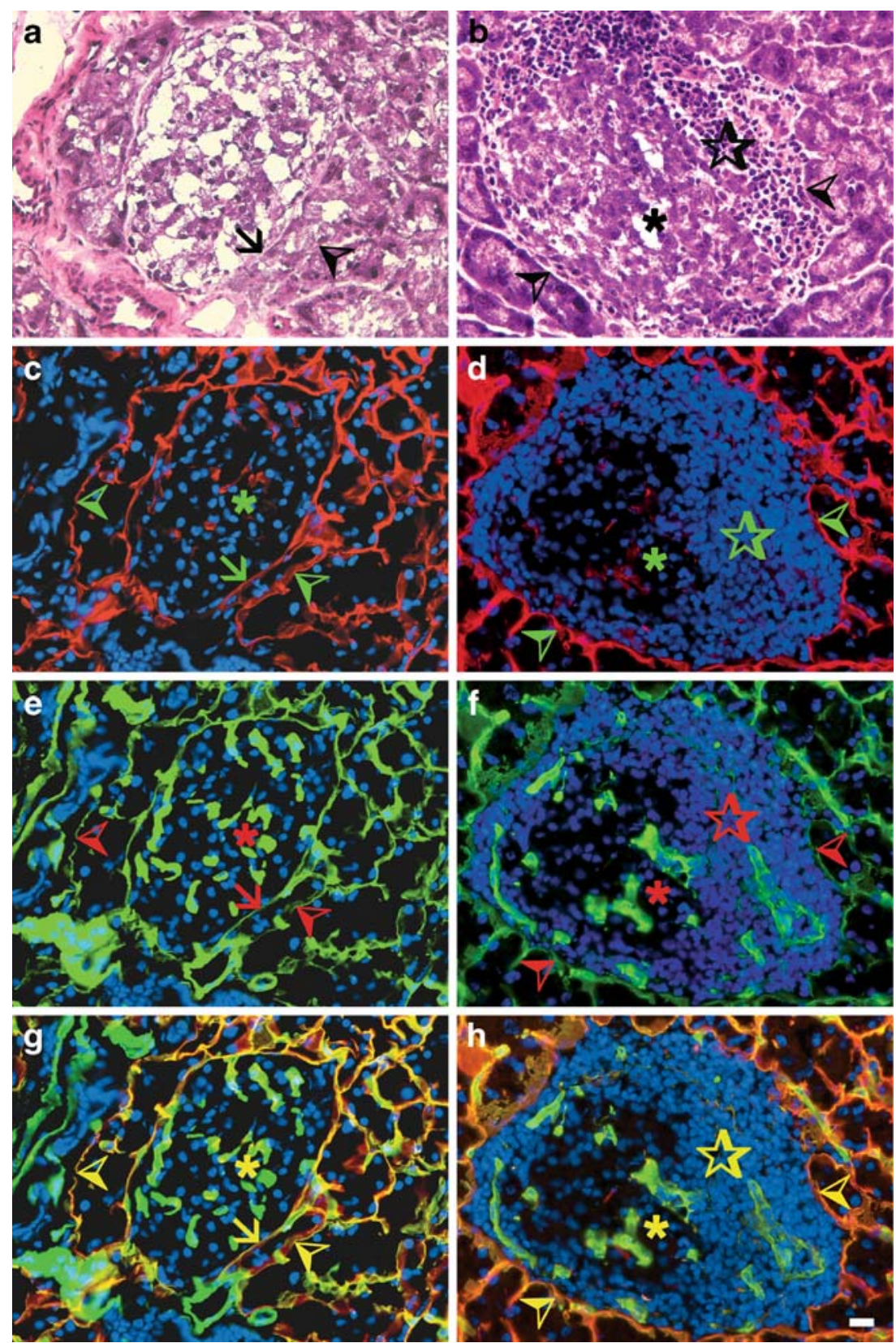

membrane exists and that, in the absence of destructive insulitis, it is a continuous structure.

Diversity in the composition of basement membranes is due to different multiple isoforms of component molecules, especially laminins and collagens type IV. This diversity provides for differences in functions between basement membranes, but can also be used to distinguish between basement membranes of different tissues and pathological states. By immunohistochemistry we observed that the periislet basement membrane specifically contains: (1) collagen type IV $\alpha 1$ and $\alpha 2$; (2) laminin chains $\alpha 2, \beta 1$ and $\gamma 1$ (suggesting that laminin 211 could be present); and (3) nidogen-1, nidogen-2 and perlecan, but not collagen type
IV $\alpha 3, \alpha 4, \alpha 5$ or $\alpha 6$. The role of the nidogens is to stabilise the collagens and laminins, while the HSPG perlecan is normally found associated with the type IV collagenlaminin backbone, generally in sub-endothelial basement membranes [16-18]. Fibronectin has been previously reported to be localised in islet basement membranes [10].

HSPGs, and perlecan in particular, have not previously been identified as components of the basement membrane of islets. This finding is significant because HSPGs are known to act as a physical barrier to cell migration or cell invasion and can provide adhesion ligands for migrating leucocytes [18]. In the context of the islet microenvironment in the NOD mouse, the presence of perlecan in the 
Fig. 3 Immunolocalisation of laminin $\beta 1$ and nidogens to the pancreases of 34.5-week-old, prediabetic NOD/Lt mice without insulitis. (a) Haematoxylin and eosin-stained section showing an islet without insulitis. (b) Section of the same islet (a), with immunolocalisation of laminin $\beta 1$ to the acinar and peri-islet basement membranes and sub-endothelial basement membranes within the islet. (c) Nidogen-1 and nidogen-2 (d) immunolocalise to the peri-islet basement membrane (arrows), sub-endothelial basement membrane (*) and basement membranes underlying acinar cells (arrowheads). (b, c, d) from different animals. Scale bar, $10 \mu \mathrm{m}$
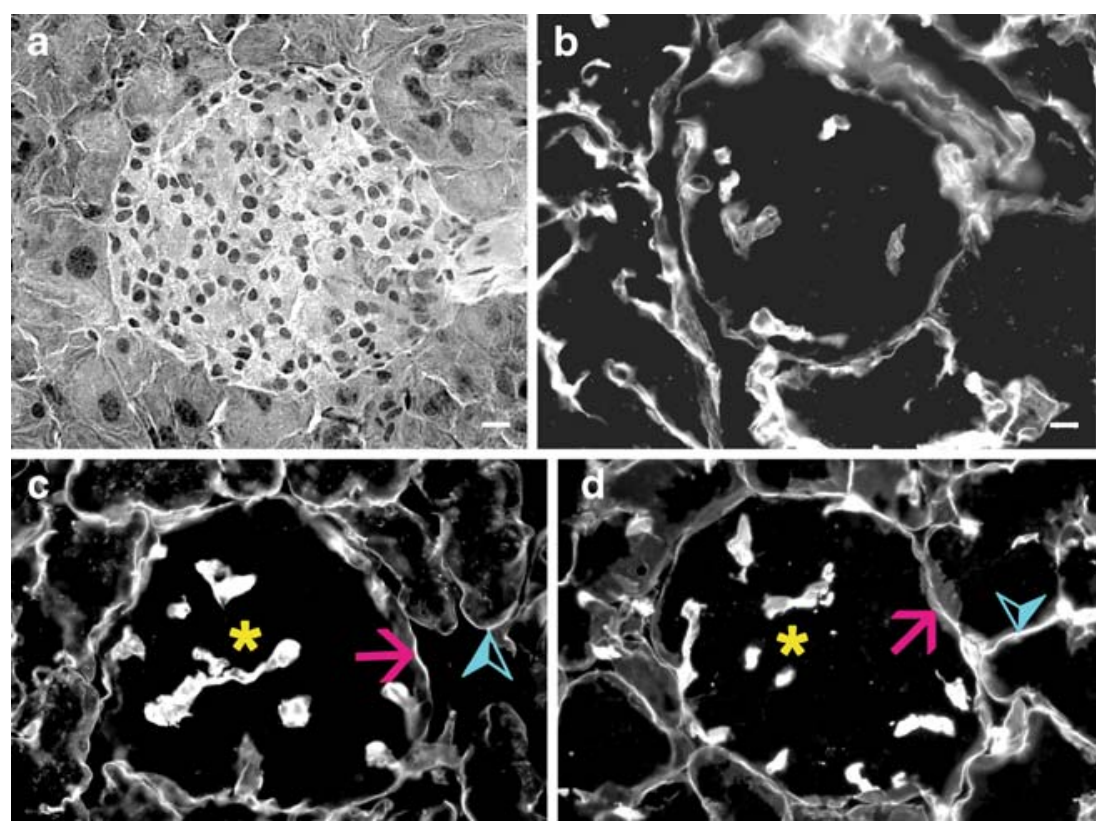

islet basement membrane could account for accumulation of insulitis MNCs around the boundary of the islets during the non-destructive phase of the autoimmune disease. Generally, perlecan provides a structural role in stabilising the interaction between other basement membrane components. The large size of HSPGs $(400-470 \mathrm{kDa})$, together with their HS side-chains, represents a barrier to metastasising tumour cells, leucocyte extravasation and leucocyte recruitment to inflammatory sites $[18,23]$.

Basement membranes are anchored to cell surfaces via integrin molecules; in particular, the $\alpha 6 \beta 1$ and $\alpha 1 \beta 1$ integrins on islet beta cells bind to laminin and collagen type IV, respectively [24]. The composition of basement membranes has been found to change during development and in disease [25], and to control the behaviour of the attached cells. Indeed via interactions with the beta cell integrins, laminin regulates insulin gene expression and beta cell replication, and collagen enhances insulin secretion $[20,24,26]$. The composition of the basement membrane can also determine whether cells can or cannot traverse. For example, in the central nervous system it has been shown that a basement membrane containing laminin
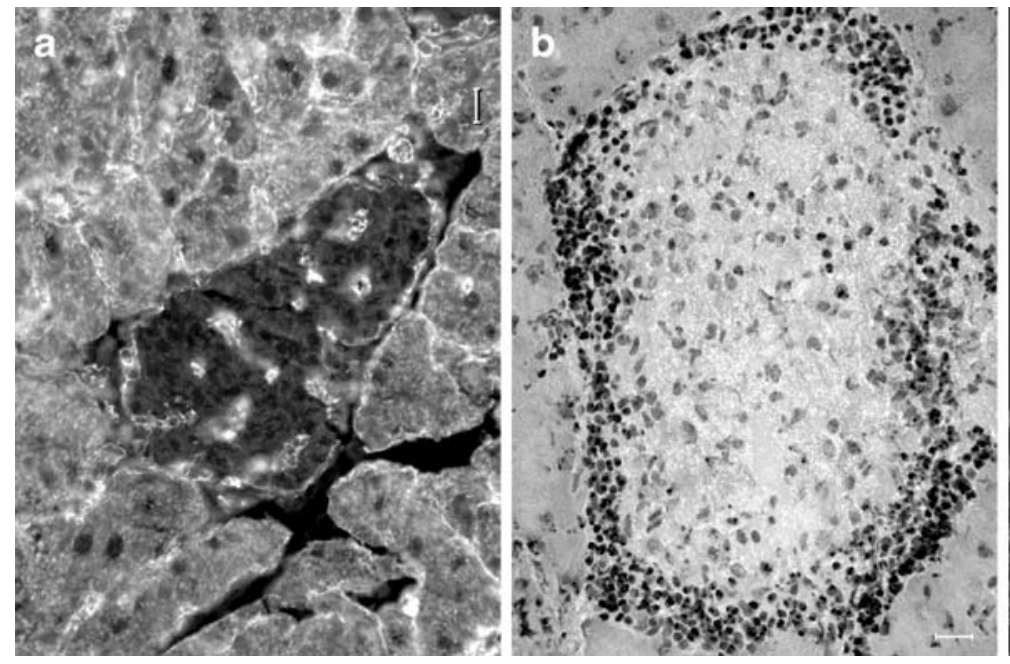

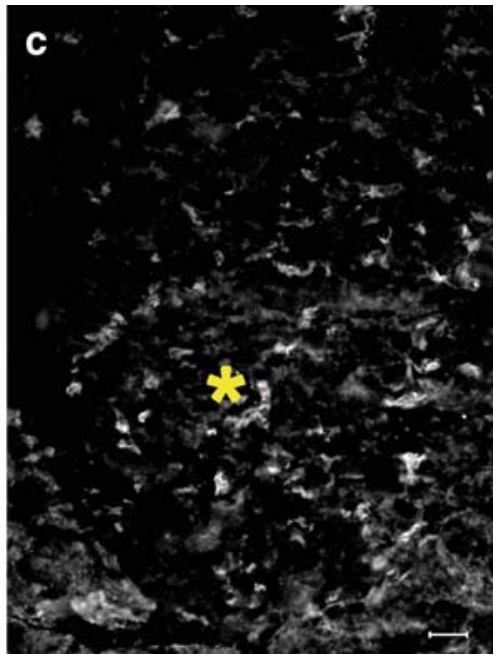

stained section showing destructive autoimmunity indicated by leucocyte infiltration around the periphery of the islet. c Section of the same islet (b). Collagen type IV is only immunolocalised to capillaries within the islet $(*)$ and not to the peri-islet basement membrane. Scale bar, $10 \mu \mathrm{m}$
Fig. 4 Localisation of collagen type IV $\alpha 1$ in pancreases of 25-weekold prediabetic NOD/Lt mice without (a) or with (c) insulitis. (a) Collagen type IV is immunolocalised to the peri-islet basement membrane, capillary sub-endothelial basement membranes and basement membranes underlying acinar cells. (b) Haematoxylin and eosin 

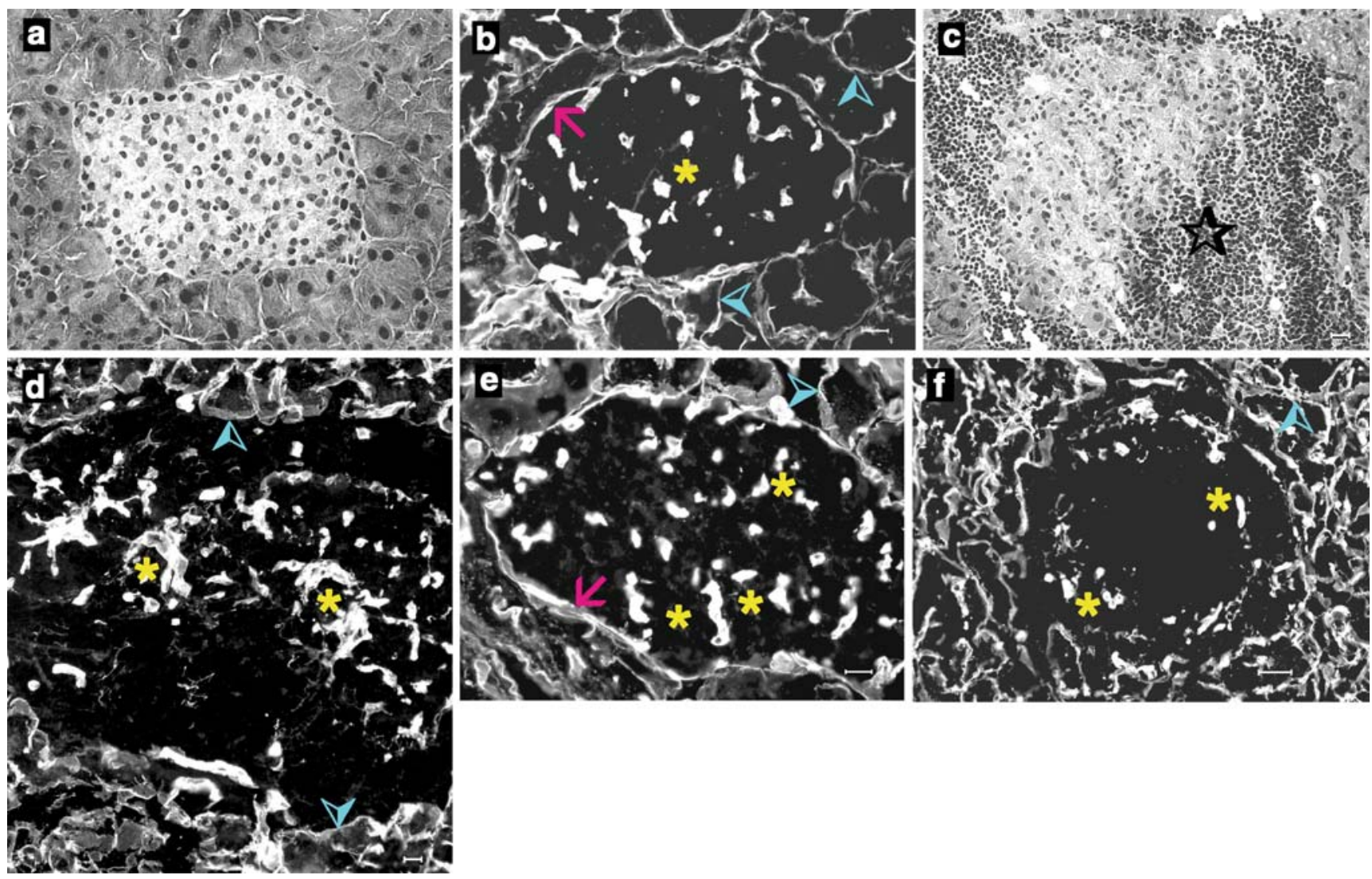

Fig. 5 Localisation of laminin $\gamma 1(\mathbf{b}, \mathbf{d})$ and perlecan $(\mathbf{e}, \mathbf{f})$ to pancreases of NOD/Lt mice with $(\mathbf{d}, \mathbf{f})$ or without $(\mathbf{b}, \mathbf{e})$ insulitis. (a) Haematoxylin and eosin-stained section, showing an islet in the pancreas of a prediabetic NOD/Lt mouse at 34.5 weeks of age. (b) Serial section of islet (a), showing laminin $\gamma 1$ immunolocalised to the peri-islet basement membrane (arrow), sub-endothelial basement membrane $(*)$ and basement membrane underlying acinar cells (arrowheads). (c) Haematoxylin and eosin stained section showing an islet in the pancreas of a prediabetic NOD/Lt mouse (34.5 weeks), with leucocyte infiltration indicating destructive autoimmunity (star). (d) Section of the same islet (c), in which laminin $\gamma 1$ localises to

$\alpha 4$ allowed T cells to cross, but a basement membrane with $\alpha 5$ did not [27]. Thus, certainty as to whether pancreatic islets have a continuous basement membrane and what that membrane is composed of is important for an understanding of the development of type 1 diabetes. No changes were observed in the composition of the peri-islet basement membrane at or after the onset of type 1 diabetes, suggesting that it was not a change in composition that initiated or allowed leucocyte infiltration of NOD islets during destructive autoimmune disease. However, islets heavily infiltrated by insulitis MNCs had no detectable components of peri-islet basement membrane, suggesting that it was completely destroyed. The most likely scenario is that extravasation of immune cells is probably from the peri-islet capillaries $[4,5]$. The lymphocytes then accumulate around the islets as a non-destructive insulitis. In other examples of inflammation, lymphocytic migration across a capillaries within the islet $(*)$ and acinar basal lamina (arrowheads), but a distinct peri-islet basement membrane is absent. (e, f) Immunolocalisation of perlecan in prediabetic NOD mouse pancreas at 25 weeks of age and in diabetic NOD/Lt mouse pancreas at 50 weeks (tissue collected on day that clinical diabetes was confirmed), respectively. Immunostaining of capillaries within the islet $(*)$ and basement membrane underlying acinar cells (arrowheads) is present in both prediabetic (e) and diabetic (f) pancreas. However, a peri-islet basement membrane (arrow) is absent in the islet from the diabetic pancreas (f). Scale bar, $10 \mu \mathrm{m}$

basement membrane requires localised destruction by degradative enzymes [18]. By analogy, the destruction of the islet basement membrane therefore needs to occur just prior to insulitis leucocyte infiltration. Clearly, the composition of the islet basement membrane will dictate the degradative enzymes needed to be produced by insulitis MNCs to permit their migration across the islet basement membrane. The islet basement membrane components identified in this study suggest that such enzymes may include heparanase (which degrades HS) and metalloproteinases (e.g. gelatinases, which break down collagen) [18, 28]. The lack of destruction of basement membranes of nearby acini and of intra-islet capillaries, which have the same components as the islet basement membrane, and the complete destruction of the islet basement membrane suggest that destruction is site-specific and localised to the immediate islet microenvironment. This injury to the islet 
basement membrane, and possibly perlecan in particular, is thus an essential process for (1) converting non-destructive autoimmunity to destructive autoimmunity, (2) the demise of islet beta cells and (3) the development of clinical symptoms of type 1 diabetes.

The mechanism of beta cell death in NOD mice is still unclear. Upon detachment from the basement membrane, epithelial cells undergo a specialised form of cell death called anoikis $[29,30]$. Similarly, destruction of the islet basement membrane could lead to programmed cell death of islet cells, a process which could contribute to the development of clinical diabetes. Transplantation studies have shown that for transplanted islets to survive, a matrix for attachment is required [11, 12]. Hence it appears that islet cell survival is dependent on interaction with the islet basement membrane and that without such an attachment islet endocrine cells die, possibly by a special pathway of apoptosis (anoikis). Our identification of the components of the peri-islet basement membrane may be of importance for developing ways to protect islets during isolation by collagenase digestion and to preserve islet integrity following transplantation.

In summary, an islet basement membrane was identified and shown to be completely destroyed by infiltrating insulitis leucocytes during the development of type 1 diabetes in NOD mice. The onset of destructive autoimmunity was not preceded by and did not correlate with a change in the composition of the basement membrane. Although the mechanism of islet basement membrane damage allowing entry of leucocytes into the intra-islet cell mass has yet to be elucidated, the islet basement membrane components identified here, and particularly the HSPG perlecan, which is known to act as a barrier to cell invasion, suggest that specialised degradative enzymes may be involved. Production of such enzymes by insulitis MNCs could represent the critical determinant in converting non-destructive autoimmunity to destructive autoimmunity and ultimately permitting the destruction of islet beta cells and the development of type 1 diabetes.

\begin{abstract}
Acknowledgements We thank S. Morris and W. Bonner for assistance with immunohistochemistry, and P. Hamilton and D. Brown for maintenance and care of the NOD/Lt mice. This research was funded by the National Health and Medical Research Council of Australia (programme grant no. 209618), The Ramaciotti Foundation, Diabetes Australia, The University of Adelaide (Adelaide, SA, Australia) and The Australian National University (Canberra, ACT, Australia).
\end{abstract}

Duality of interest The authors declare that there is no duality of interest associated with this manuscript.

Open Access This article is distributed under the terms of the Creative Commons Attribution Noncommercial License which permits any noncommercial use, distribution, and reproduction in any medium, provided the original author(s) and source are credited.

\section{References}

1. Jansen A, Homo-Delarche F, Hooijkaas H, Leenen PJ, Dardenne M, Drexhage HA (1994) Immunohistochemical characterization of monocytes-macrophages and dendritic cells involved in the initiation of the insulitis and beta-cell destruction in NOD mice. Diabetes 43:667-675

2. Solomon M, Sarvetnick N (2004) The pathogenesis of diabetes in the NOD mouse. Adv Immunol 84:239-264

3. Mathis D, Vence L, Benoist C (2001) beta-Cell death during progression to diabetes. Nature 414:792-798

4. Papaccio G, Latronico MV, Pisanti FA, Federlin K, Linn T (1998) Adhesion molecules and microvascular changes in the nonobese diabetic (NOD) mouse pancreas. An NO-inhibitor (L-NAME) is unable to block adhesion inflammation-induced activation. Autoimmunity 27:65-77

5. Papaccio G, Pisanti FA, Montefiano RD, Graziano A, Latronico MV (2002) Th1 and Th2 cytokines exert regulatory effects upon islet microvascular areas in the NOD mouse. J Cell Biochem 86:651-664

6. van Deijnen JH, Hulstaert CE, Wolters GH, van Schilfgaarde R (1992) Significance of the peri-insular extracellular matrix for islet isolation from the pancreas of rat, dog, pig, and man. Cell Tissue Res 267:139-146

7. Jiang FX, Naselli G, Harrison LC (2002) Distinct distribution of laminin and its integrin receptors in the pancreas. J Histochem Cytochem 50:1625-1632

8. Miner JH, Li C, Patton BL (2004) Laminins alpha2 and alpha4 in pancreatic acinar basement membranes are required for basal receptor localization. J Histochem Cytochem 52:153-156

9. Pavin EJ, Pinto GA, Zollner RL, Vassallo J (2003) Immunohistochemical study of the pancreatic basement membrane in non obese diabetic mice (NOD) with spontaneous autoimmune insulitis. J Submicrosc Cytol Pathol 35:25-27

10. Geutskens SB, Homo-Delarche F, Pleau JM, Durant S, Drexhage HA, Savino W (2004) Extracellular matrix distribution and islet morphology in the early postnatal pancreas: anomalies in the nonobese diabetic mouse. Cell Tissue Res 318:579-589

11. Thomas FT, Contreras JL, Bilbao G, Ricordi C, Curiel D, Thomas JM (1999) Anoikis, extracellular matrix, and apoptosis factors in isolated cell transplantation. Surgery 126:299-304

12. Wang RN, Rosenberg L (1999) Maintenance of beta-cell function and survival following islet isolation requires re-establishment of the islet-matrix relationship. J Endocrinol 163:181-190

13. Irving-Rodgers HF, Bathgate RA, Ivell R, Domagalski R, Rodgers RJ (2002) Dynamic changes in the expression of relaxin-like factor (INSL3), cholesterol side-chain cleavage cytochrome $\mathrm{p} 450$, and 3 beta-hydroxysteroid dehydrogenase in bovine ovarian follicles during growth and atresia. Biol Reprod 66:934-943

14. Iozzo RV (2005) Basement membrane proteoglycans: from cellar to ceiling. Nat Rev Mol Cell Biol 6:646-656

15. Virtanen I, Banerjee M, Palgi J et al (2008) Blood vessels of human islets of Langerhans are surrounded by a double basement membrane. Diabetologia 51:1181-1191

16. Paulsson M (1992) Basement membrane proteins: structure, assembly, and cellular interactions. Crit Rev Biochem Mol Biol 27:93-127

17. Timpl R, Brown JC (1996) Supramolecular assembly of basement membranes. Bioessays 18:123-132

18. Parish CR (2006) The role of heparan sulphate in inflammation. Nat Rev Immunol 6:633-643

19. Wang R, Li J, Rosenberg L (2001) Factors mediating the transdifferentiation of islets of Langerhans to duct epithelial-like structures. J Endocrinol 171:309-318 
20. Nikolova G, Jabs N, Konstantinova I et al (2006) The vascular basement membrane: a niche for insulin gene expression and beta cell proliferation. Dev Cell 10:397-405

21. Kilkenny DM, Rocheleau JV (2008) Fibroblast growth factor receptor-1 signaling in pancreatic islet beta-cells is modulated by the extracellular matrix. Mol Endocrinol 22:196-205

22. Wang RN, Paraskevas S, Rosenberg L (1999) Characterization of integrin expression in islets isolated from hamster, canine, porcine, and human pancreas. J Histochem Cytochem 47:499-506

23. Parish CR, Freeman C, Hulett MD (2001) Heparanase: a key enzyme involved in cell invasion. Biochim Biophys Acta 1471:M99-M108

24. Nikolova G, Strilic B, Lammert E (2007) The vascular niche and its basement membrane. Trends Cell Biol 17:19-25

25. Yurchenco PD, Amenta PS, Patton BL (2004) Basement membrane assembly, stability and activities observed through a developmental lens. Matrix Biol 22:521-538

26. Kaido T, Yebra M, Cirulli V, Montgomery AM (2004) Regulation of human beta-cell adhesion, motility, and insulin secretion by collagen IV and its receptor alphalbeta1. J Biol Chem 279:53762-53769
27. Sixt M, Engelhardt B, Pausch F, Hallmann R, Wendler O, Sorokin LM (2001) Endothelial cell laminin isoforms, laminins 8 and 10, play decisive roles in T cell recruitment across the blood-brain barrier in experimental autoimmune encephalomyelitis. J Cell Biol 153:933-946

28. Yadav R, Larbi KY, Young RE, Nourshargh S (2003) Migration of leukocytes through the vessel wall and beyond. Thromb Haemost 90:598-606

29. Grossmann J (2002) Molecular mechanisms of 'detachmentinduced apoptosis-Anoikis'. Apoptosis 7:247-260

30. Zvibel I, Smets F, Soriano H (2002) Anoikis: roadblock to cell transplantation. Cell Transplant 11:621-630

31. Sado Y, Kagawa M, Kishiro Y et al (1995) Establishment by the rat lymph node method of epitope-defined monoclonal antibodies recognizing the six different alpha chains of human type IV collagen. Histochem Cell Biol 104:267-275

32. Dziadek M, Paulsson M, Timpl R (1985) Identification and interaction repertoire of large forms of the basement membrane protein nidogen. Embo J 4:2513-2518 